\title{
SAFETY AND SKIN DELAYED-TYPE HYPERSENSITIVITY RESPONSE IN VERVET MONKEYS IMMUNIZED WITH Leishmania donovani SONICATE ANTIGEN DELIVERED WITH ADJUVANTS
}

\author{
Joshua M. MUTISO(1,3), John C. MACHARIA(1), Evans TARACHA(1), Kellern WAFULA(2), Hitler RIKOI(1) \& Michael M. GICHERU(3)
}

\begin{abstract}
SUMMARY
In this study, we report on the safety and skin delayed-type hypersensitivity (DTH), responses of the Leishmania donovani whole cell sonicate antigen delivered in conjunction with alum-BCG (AlBCG), Montanide ISA 720 (MISA) or Monophosphoryl lipid A (MPLA) in groups of vervet monkeys. Following three intradermal injections of the inoculums on days 0,28 and 42, safety and DTH responses were assessed. Preliminary tumor necrosis factor alpha (TNF- $\alpha$ ) and interferon gamma (IFN- $\gamma$ ) levels were also measured and these were compared with DTH. Only those animals immunized with alum-BCG reacted adversely to the inoculum by producing ulcerative erythematous skin indurations. Non-parametric analysis of variance followed by a post-test showed significantly higher DTH responses in the MISA+Ag group compared with other immunized groups $(\mathrm{p}<0.001)$. The MPLA+Ag group indicated significantly lower DTH responses to the sonicate antigen compared with the AlBCG+Ag group. There was a significant correlation between the DTH and cytokine responses $(p<0.0001)$. Based on this study we conclude that Leishmania donovani sonicate antigen containing MISA 720 is safe and is associated with a strong DTH reaction following immunization.
\end{abstract}

KEYWORDS: Safety; Delayed-type hypersensitivity (DTH); Vervet monkeys; Leishmania donovani sonicate antigen; Adjuvants.

\section{INTRODUCTION}

Visceral leishmaniasis (VL) or kala-azar is the most dreaded and devastating amongst the various forms of leishmaniasis (GARG \& DUBE, 2006). The disease is a major cause of morbidity and mortality in East Africa and the Indian subcontinent. Coinfection with HIV enhances the risk of the disease (NGURE et al., 2009). The only control measures currently available in East Africa are case detection and treatment with antimonial drugs, which are expensive, toxic, not always available and cannot be self-administered. Vector and reservoir control is difficult due to the elusive nature of the vector and the diversity of the animal reservoir. Cases of self-cure in cutaneous leishmaniasis, accompanied by solid immunity to reinfection, make vaccine development a feasible control method (MUTISO et al., 2010a). Cellular or Th1 immune response is important for the cure of leishmaniasis. This involves the activation of infected macrophages by sensitized $\mathrm{T}$ cells to produce parasite lethal nitric oxide. The use of adjuvants in effective Leishmania vaccine development cannot be avoided. The availability of many potential adjuvants has prompted the need for identifying rational standards for the selection of adjuvant formulations based on safety and sound immunological principles for human vaccines (ALVING, 2002). Three effective adjuvants that have shown promising results in Leishmania vaccine studies have been identified in a recent review (MUTISO et al., 2010a). These adjuvants include: Montanide ISA 720 (MUTISO et al., 2010b), alum formulated with BCG (MISRA et al., 2001; KAMIL et al., 2003) and Monophosphoryl lipid A (SKEIKY et al., 2002; COLER \& REED, 2005; COLER et al., 2007). Despite a lot of vaccination research using these adjuvants, it is not readily clear which of them is more superior in terms of safety and immunogenicity and hence which of them is most appropriate for use in the development of Leishmania vaccines. Skin delayed-type hypersensitivity (DTH) response has been used as an indicator of the immunogenicity in an immunization antigen measured by the presence of a specific cellular type of immune reaction (GICHERU et al., 2001; RITTER \& KORNER, 2002; MASINA et al., 2003). The present report describes a study undertaken to evaluate the safety and DTH response of Leishmania donovani sonicate antigen delivered together with each of the three selected adjuvants in the vervet monkey model with visceral leishmaniasis. The study also measured and compared preliminary TNF- $\alpha$ and IFN- $\gamma$ levels with the DTH response.

\section{MATERIALS AND METHODS}

Leishmania parasites: The Leishmania donovani strain NLB-065 originated from the spleen of an infected patient in the Baringo district of Kenya and was maintained by intracardiac hamster-to-hamster passage at the Institute of Primate Research. A hamster splenic biopsy was cultured in Schneider's drosophila insect medium and was supplemented 


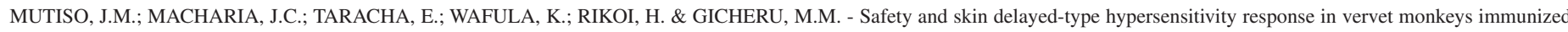
with Leishmania donovani sonicate antigen delivered with adjuvants. Rev. Inst. Med. Trop. Sao Paulo, 54(1): 37-41, 2012.

with $20 \%$ fetal bovine serum and $100 \mu \mathrm{g} / \mathrm{mL}$ of gentamicin at $25^{\circ} \mathrm{C}$ till the stationary phase. Stationary phase promastigotes were harvested at $2500 \mathrm{rpm}$ (Servoll 6000D) for $15 \mathrm{~min}$ at $4{ }^{\circ} \mathrm{C}$. The pellets were washed three times in sterile phosphate buffered saline (PBS) by centrifugation. These parasites were used for antigen preparation, immunization and DTH assessment.

Adjuvants and vaccine preparation: Monophosphoryl lipid A (InvivoGen), Montanide ISA 720 V (Seppic), alum (Rehydragel HPA; Reheis, Berkeley Heights, NJ) and BCG (Serum Institute of India, Hadapsar, India) were used as adjuvants in this study. The immunization antigen was prepared from Leishmania donovani promastigotes. Stationary phase promastigotes were harvested as previously described, counted and resuspended in $3 \mathrm{~mL}$ PBS at a concentration of $8 \times 10^{8}$ promastigotes. These promastigotes were frozen - thawed three times in liquid nitrogen and sonicated at $18 \mathrm{kHz}$ for five periods of 45 seconds each on ice, separated by one minute intervals. Each dose of the immunization and DTH assessment antigen was prepared from $1 \times 10^{7}$ promastigotes equivalent to $100 \mu \mathrm{g}$ of Leishmania donovani sonicate antigen.

Vervet monkeys: Both young and adult vervet monkeys of both sexes were caught in the wild and quarantined for 120 days at the Institute of Primate Research, Karen, Nairobi, Kenya. During the quarantine period, the monkeys were monitored for Mycobacterium tuberculosis infection and gastrointestinal and parasitic infections. The animals were tested for antileishmanial antibodies against both Leishmania donovani and $L$. major antigen by ELISA and monkeys with no antibody titer were selected for the study. These animals were housed individually in squeeze-back cages and fed a commercial non-human primate meal, supplemented weekly with fruits and vegetables. Water was provided $a d$ libitum. Institutional Animal Care and Use and Institutional Scientific resources and Evaluation Committee guidelines were strictly followed.

Experimental protocol: Leishmania donovani antibody-free adult vervet monkeys with a mean body weight of $3.4 \mathrm{~kg}$ were selected and divided into five groups of three monkeys each as follows: Group 1, alum precipitated sonicate plus BCG (AlBCG+Ag); Group 2, sonicate plus monophosphoryl lipid A (MPLA+Ag); Group 3, sonicate plus montanide ISA 720 (MISA+Ag); Group 4, sonicate (Ag) alone and Group 5 non-vaccinated control (naïve control). The experimental groups were vaccinated three times intradermally on days 0,28 , and 42 . Between days 0 and 60 vaccinated animals were monitored for safety parameters. The skin delayed-type hypersensitivity responses were assessed on day 63 following 72 hours of intradermal antigen inoculation. The DTH results were compared with preliminary TNF- $\alpha$ and IFN- $\gamma$ levels (measured on day 63 following initial vaccination) to ascertain the usefulness of using DTH as a predictive marker of Th1 immune responses in immunized subjects.

Assessment of safety: The animals were observed for any induration and erythema at the sites of vaccine injection. Examination of the regional lymphadenopathy was also carried out. Body temperature and weight changes were also monitored.

Assessment of skin delayed-type hypersensitivity (DTH): Three weeks after the third vaccination, animals were assessed for DTH responses by intradermal inoculation with $100 \mu \mathrm{g}$ of $L$. donovani antigen, equivalent to sonicated Leishmania donovani promastigotes in $100 \mu \mathrm{L}$ of PBS. Seventy two hours later, skin indurations at the inoculation sites were measured using a metric caliper as described (GICHERU et al., 2001). A mean induration diameter $>5 \mathrm{~mm}$ was considered to be positive.

Quantification of cytokines: Tumor necrosis factor alpha and IFN- $\gamma$ were measured in cell-culture supernatants. Peripheral blood mononuclear cells (PBMCs) were isolated from the whole blood by density gradient centrifugation as described (OLOBO et al., 1990). Purified cells were adjusted to $3 \times 10^{6} / \mathrm{mL}$ in a complete RPMI 1640 medium and stimulated with Leishmania donovani antigen as previously described (GICHERU et al., 1995). Cultures supernatants were collected from triplicate wells after 72 hours of stimulation and the concentration of TNF- $\alpha$ and IFN- $\gamma$ in the supernatant was determined by enzyme linked immunosorbent assay (ELISA). Polystyrene flat bottomed micro-ELISA plates (Dynatech Laboratories, Sussex, UK) were briefly coated overnight with $50 \mu \mathrm{L}$ of $2 \mu \mathrm{g} / \mathrm{mL}$ of capture monoclonal antibodies (Becton Dickinson and MabTech, Sweden) diluted in bicarbonate buffer, pH 9.6. Excess coating buffer was removed, and non-specific binding sites were blocked in 3\% bovine serum albumin (Sigma) in PBS for one hour at $37^{\circ} \mathrm{C}$. The plates were washed four times with $0.005 \%$ Tween 20 in PBS, and $50 \mu \mathrm{L}$ of culture supernatant was dispensed to the appropriate wells. Human TNF- $\alpha$ (Becton Dickinson) and IFN- $\gamma$ diluted (1 to $600 \mathrm{U} / \mathrm{mL}$ ) in $1 \%$ bovine serum albumin in PBS-Tween were used as standards. The plates were incubated at $37^{\circ} \mathrm{C}$ for one hour and washed four times before the addition of biotinylated secondary monoclonal antibodies ( $50 \mu \mathrm{L}$ of $1 / 2000$ dilution) followed by incubation at $37^{\circ} \mathrm{C}$ for one hour. The plates were washed four times as before, $50 \mu \mathrm{L}$ of $1 / 300$-diluted alkaline phosphate-conjugated streptavidin was added, and the mixtures were incubated for one hour as described above. The plates were washed ten times in PBS-Tween, and $50 \mu \mathrm{L}$ of nitrophenyl phosphate substrate $(1 \mathrm{mg} / \mathrm{mL})$ in diethanolamine buffer was added. The plate was incubated at $37^{\circ} \mathrm{C}$ in the dark for 30 minutes, and absorbance was read at $405 \mathrm{~nm}$.

Statistical analysis: Non-parametric one-way analysis of variance (ANOVA) was used to compare the means of groups. The Tukey-Kramer test was used for inter-group statistical analysis. Differences were considered significant where $p<0.05$. Spearman rank correlation was used for correlation analysis.

\section{RESULTS}

Local reaction to Leishmania donovani sonicate antigen-adjuvant immunization: All animals in the AlBCG+Ag group showed indurations at the sites of vaccination. These indurations, measured seven days following immunizations, lasted between 34 and 41 days before complete resolution. These indurations were ulcerative and associated with erythema. Lymph node swellings were also observed in the AlBCG+Ag group and these resolved with the disappearance of indurations. Animals in other groups did not show any lymphadenopathy or local skin reaction to the immunizations. There were no systemic side-effects such as fever or weight loss in any of the animal groups following immunization.

Delayed-type hypersensitivity response: All immunized monkeys developed DTH response to Leishmania donovani antigen as measured on day 21 after the second immunization booster. Post-test analysis indicated a significantly higher DTH reaction in the MISA+Ag group compared with each of the other groups $(p<0.001)$. The AlBCG+Ag group showed a significantly higher DTH response to the sonicate antigen 


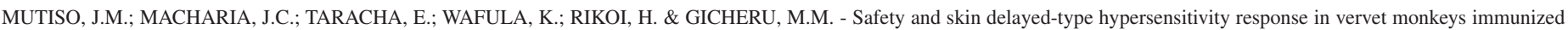
with Leishmania donovani sonicate antigen delivered with adjuvants. Rev. Inst. Med. Trop. Sao Paulo, 54(1): 37-41, 2012.

compared with the MPLA+Ag and Ag alone groups as well as the naïve control group $(p<0.001)$. It was interesting to note that there was no difference in DTH responses between the MPLA+Ag and the Ag alone groups $(p>0.05)$. The naïve control animals did not exhibit a positive DTH response as shown in Figure 1.

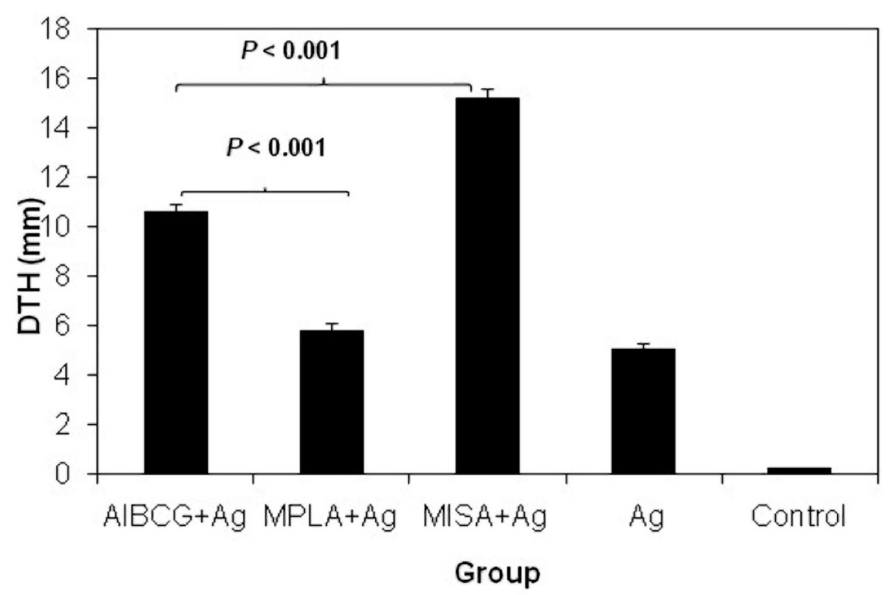

Fig. 1 - Skin delayed-type hypersensitivity (DTH) responses in vervet monkeys following immunizations. Animals were immunized at three different points in time with sonicate antigen $(\mathrm{Ag})$ alone; sonicate antigen plus alum plus BCG (AlBCG+Ag); sonicate antigen plus monophosphoryl lipid A (MPLA+Ag) or sonicate plus montanide ISA $720 \mathrm{~V}$ (MISA+Ag) and the DTH determined on day 63 following initial immunization. Data shown indicates the mean DTH \pm SD in each immunization group. A DTH value $<5 \mathrm{~mm}$ was considered negative.

Th1 cytokine responses: $\mathrm{B}$ oth $\mathrm{AlBCG}+\mathrm{Ag}$ and $\mathrm{MISA}+\mathrm{Ag}$ vaccinated groups induced comparable TNF- $\alpha$ cytokine levels $(p>$ $0.05)$ which were significantly higher $(p<0.05)$ than the levels produced by the MPLA+Ag group. Although the MPLA+Ag immunized group induced significantly higher $(p<0.05)$ TNF- $\alpha$ levels than the control group, it was interesting to note that the cytokine levels induced in both $\mathrm{Ag}$ and MPLA+Ag groups were comparable ( $p>0.05$; Fig. 2). This trend of TNF- $\alpha$ production was similar to the IFN- $\gamma$ production except that, MPLA+Ag induced significantly higher levels of IFN- $\gamma$ compared with the Ag group (data not shown).

Relationship between DTH and Th1 cytokine responses: Spearman rank correlation analysis indicated a significant correlation between DTH responses and corresponding TNF- $\alpha$ values $(r=0.9374$; $p<0.0001)$. A positive and significant correlation was also established between DTH and IFN- $\gamma$ responses $(r=0.9410 ; p<0.0001$; Fig. 3$)$.

\section{DISCUSSION}

Visceral leishmaniasis is a major health problem in East Africa where it is associated with frequent epidemics that claim hundreds of lives, especially in the remote areas where medical care facilities are limited. Available drugs are very expensive, and highly toxic. The development of a safe vaccine may provide an immunological control strategy. There is no available vaccine for the control of leishmaniasis to date (MUTISO et al., 2010b). It is apparent that an effective Leishmania vaccine will only be achieved by careful selection of both an appropriate adjuvant as well as an antigen (MUTISO et al., 2010a). Hundreds of different adjuvants exist, and these need to be selected on the basis of their safety and sound

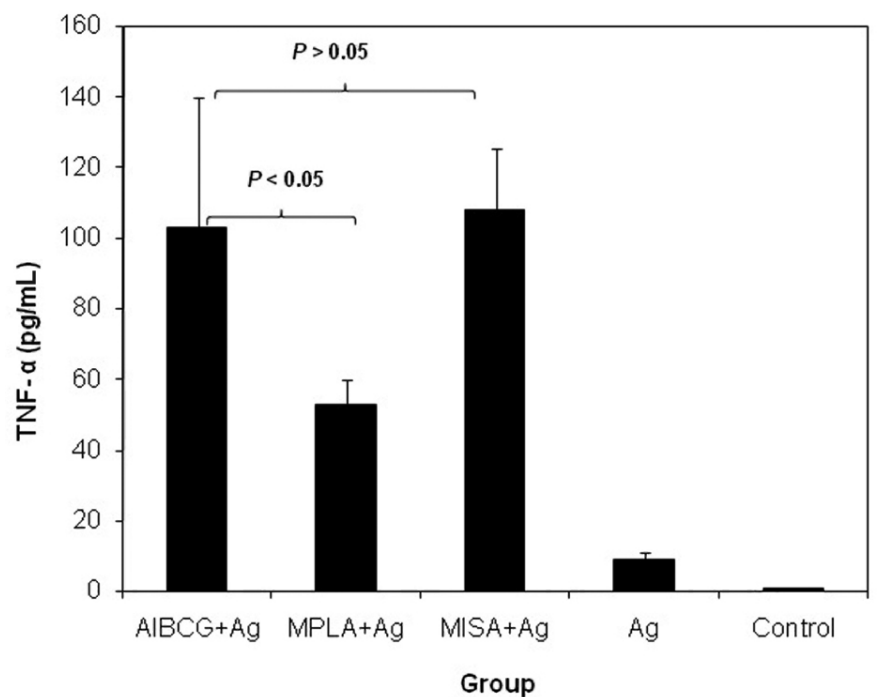

Fig. 2 - Level of TNF- $\alpha$ response to Leishmania donovani antigen in vervet monkeys vaccinated at three different points in time with sonicate antigen $(\mathrm{Ag})$ alone; or in conjunction with alum plus BCG (AlBCG+Ag); monophosphoryl lipid A (MPLA+Ag) or montanide ISA $720 \mathrm{~V}$ (MISA+Ag). The values for the antigen-adjuvant vaccinated animals on day 63 following initial vaccination were comparable with AlBCG+Ag and MISA+Ag and significantly higher $(p<0.05)$ than with the MPLA+Ag group.

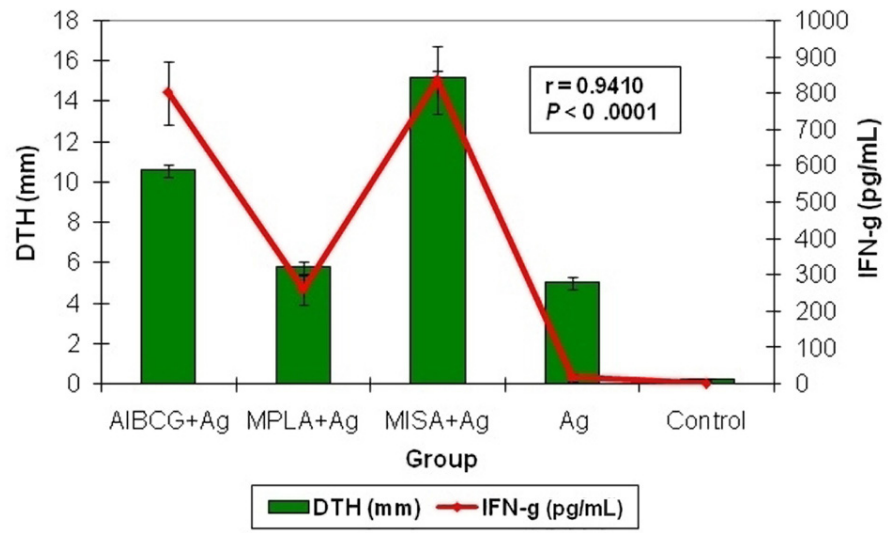

Fig. 3 - Relationship between delayed-type hypersensitivity (DTH) skin and IFN- $\gamma$. Animals were immunized at three different points in time with sonicate antigen (Ag) alone; or in conjunction with alum plus BCG (AlBCG+Ag); monophosphoryl lipid A (MPLA+Ag) or montanide ISA $720 \mathrm{~V}$ (MISA+Ag) and the DTH and IFN- $\gamma$ determined on day 63 following initial immunization and a correlation analysis between DTH and IFN- $\gamma$ was carried out. A DTH value of $5 \mathrm{~mm}$ and above was considered positive. Data shown indicates a positive and significant correlation between DTH and IFN- $\gamma$ responses.

immunological principles. Promising adjuvants include Montanide ISA 720 MUTISO et al., 2010b), alum/BCG (MISRA et al., 2001; KAMIL et al., 2003) and Monophosphoryl lipid A (COLER et al., 2007). These adjuvants have been associated with Th1 cytokine as well as humoral immune responses. It is not yet clear which of these adjuvants would be superior in inducing a cellular immune response required for the control of leishmaniasis. Positive DTH response is a marker of a type 1 immune response and has been used to assess the immunogenicity of candidate vaccine antigens against leishmaniasis (MISRA et al., 2001; GICHERU et al., 2001; MASINA et al., 2003). This study set out to identify the most important adjuvant for the development of leishmania 


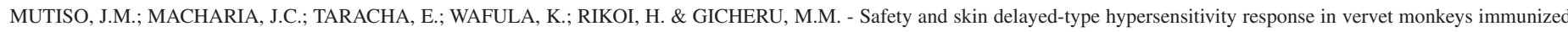
with Leishmania donovani sonicate antigen delivered with adjuvants. Rev. Inst. Med. Trop. Sao Paulo, 54(1): 37-41, 2012.

vaccines. Parameters assessed included safety and immunogenicity based on positive DTH and Th1 cytokine (TNF- $\alpha$ and IFN- $\gamma$ ). A recent study carried out in our laboratory indicated montanide ISA 720 as a better adjuvant than BCG for leishmania vaccines in mice (MUTISO et al., 2010b). The study recommended confirmation of these results in a non-human primate model of the disease.

The results of our present study show that based on two clinical parameters including fever and changes in body weight, the immunization protocols are safe. Following initial immunization, daily observation of immunized animals was carried out and we did not observe any local or systemic side effects in any of the animals immunized with MPLA+Ag or MISA+Ag. The safety of MISA has been reported elsewhere including in vaccination studies carried out in our laboratory (TOLEDO et al., 2001; MASINA et al., 2003; OLIVEIRA et al., 2005). Our safety report on MISA as an adjuvant for Leishmania vaccine differs from other studies that have associated this adjuvant with adverse reactions including swelling and erythema at the injection site observed within a few days following vaccination (PIERCE et al., 2010). Other studies have reported occasional unacceptable local reactogenicity in vaccines containing montanides (WU et al., 2008). It is likely that adverse reactions observed following immunizations with vaccines containing MISA may be a result of the immunizing antigen. Monophosphoryl lipid A has also been found to be safe in a vaccine study performed on healthy human toddlers (VERNACCHIO et al., 2002). Earlier studies had also indicated the safety and effectiveness of MPLA in Leishmania vaccine studies (REED et al., 2003). The additional safety evaluation of MPLA as an adjuvant for clinical trials had earlier been performed on dogs, rabbits and rats (BALDRICK et al., 2002). However, as a matter of serious concern, the study has established that, based on adverse local skin reaction, an immunization containing BCG is not safe as it is associated with erythematous ulcerative skin nodules or indurations. This confirms the results observed in our earlier study on mice (MUTISO et al., 2010b) where vaccination with BCG was associated with adverse reaction. Other studies involving the use of the monkey system have also associated BCG with severe reactions (MISRA et al., 2001). Lymphadenopathy was also an undesirable feature in the use of BCG as an adjuvant in this study. Delayed-type hypersensitivity response to the Leishmania donovani antigen was prominent in the animal group immunized with sonicate plus MISA, an indication that this adjuvant may be important for the development of a safe and effective vaccine against visceral leishmaniasis. Remarkable levels of TNF- $\alpha$ and IFN- $\gamma$ induced by groups vaccinated with antigen containing Alum-BCG and MISA indicates the superiority of these adjuvants in the selective induction of Th1 immune responses. Alum-BCG has been indicated to induce strong Th1 immune responses in Leishmania vaccine studies (MISRA et al., 2001; KAMIL et al., 2003). In malaria, HIV and cancer vaccine studies, MISA 720 has been indicated to induce high Th1 immune responses important for the control of these diseases (KENNEY \& ELDELMAN, 2003). This adjuvant has also been shown to induce strong Th1 cytokine in Leishmania vaccination studies (MASINA et al., 2003; MUTISO et al., 2010b). The strong correlation between DTH and both TNF- $\alpha$ and IFN- $\gamma$ responses in the present study indicates the importance of the DTH response as an early marker of the outcome of a vaccination study. It may be possible that, very expensive experimental vaccination studies may be designed to predict field trial outcome by preliminary determination of DTH responses. However, optimization studies need to be carried out to validate this observation. Although immunization with $\mathrm{AlBCG}+\mathrm{Ag}$ and MISA 720 induced comparable and significantly higher DTH and Th1 cytokine responses compared with the MPLA+Ag immunization, the association of BCG with adverse skin reaction may preclude it from future evaluation for possible use in the development of a safe vaccine in humans. Monophosphoryl lipid A has been indicated as an adjuvant with desirable results in vaccination studies against murine leishmaniasis (COLER et al., 2007). In the present study, the failure of the MPLA+Ag immunized group to induce a higher DTH reaction than the Ag alone group may be a factor in the MPLA formulation. COLER et al. (2007) used the oil based MPL-ES (MPL-A in stable emulsion) while our current study used MPL-A based on aqueous formulation. Furthermore the MPLA used in this study, a natural product, is a heterogeneous mixture, which may affect its effectiveness (COLER et al., 2009).

We conclude that, the intradermally injected Leishmania donovani sonicate antigen plus montanide ISA 720 was safe and that the rate of skin delayed-type hypersensitivity conversion is higher than that induced by AlBCG+Ag or MPLA+Ag. In addition, although both MISA+Ag and $\mathrm{AlBCG}+\mathrm{Ag}$ induce desirable TNF- $\alpha$ and IFN- $\gamma$ responses, the association of AlBCG with unacceptable adverse reaction leaves montanide ISA 720 $\mathrm{V}$ as a potentially superior adjuvant for candidate vaccine for visceral leishmaniasis. However, further studies are necessary to compare the immunogenicity between montanide ISA 720 and monophosphoryl lipid A formulated in a stable emulsion.

\section{RESUMO}

Segurança e reação de hipersensibilidade tardia na pele de macacos vervet imunizados com antígeno sonicado de Leishmania donovani junto com adjuvantes

Neste estudo reportamos segurança e resposta de hipersensibilidade tardia (DTH) do antígeno sonicado de células totais de Leishmania donovani introduzidos juntamente com alume-BCG (AIBCG) Montanide ISA 720 (MISA) ou lípide A monofosforilado (MPLA) em grupos de macacos vervet. Depois de três injeções intradérmicas do inóculo nos dias 0,28 e 42 segurança e resposta DTH foram avaliados. Preliminarmente níveis de fator de necrose tumoral alfa (TNF- $\alpha)$ e interferon gama (IFN- $\gamma$ ) foram também medidos e comparados com o DTH. Somente os animais imunizados com alume-BCG reagiram de maneira diversa ao inóculo produzindo indurações ulceradas e eritematosas na pele. Análise não paramétrica de variação seguida por um teste posterior mostraram resposta significantemente mais alta do DTH no grupo MISA + Ag quando comparado com outros grupos imunizados $(p<0.001)$. $\mathrm{O}$ grupo MPLA + Ag demonstrou resposta DTH significantemente menor do antígeno sonicado comparado com o grupo AIBCG + Ag. Houve correlação significante entre o DTH e a resposta às citocinas ( $p$ $<0.0001$ ). Baseados neste estudo concluímos que o antígeno sonicado de Leishmania donovani contendo MISA 720 é seguro e está associado com forte reação DTH após imunização.

\section{ACKNOWLEDGEMENTS}

This study was supported by a grant from the National Council for Science and Technology (NCST; Grant code: NCST/5/003/CALL2/266) of the Government of Kenya. We express our gratitude to SEPPIC, France through Dr Amina Bensaber for generously providing us with the Montanide ISA 720 adjuvant used in this study. 
MUTISO, J.M.; MACHARIA, J.C.; TARACHA, E.; WAFULA, K.; RIKOI, H. \& GICHERU, M.M. - Safety and skin delayed-type hypersensitivity response in vervet monkeys immunized with Leishmania donovani sonicate antigen delivered with adjuvants. Rev. Inst. Med. Trop. Sao Paulo, 54(1): 37-41, 2012.

\section{REFERENCES}

1. Alving CR. Design and selection of vaccine adjuvants: animal models and human trials. Vaccine. 2002;20(Suppl 3):S56-64.

2. Baldrick P, Richardson D, Elliott G, Wheeler AW. Safety evaluation of monophosphoryl lipid A (MPL): an immunostimulatory adjuvant. Regul Toxicol Pharmacol. 2002;35:398-413.

3. Coler RN, Carter D, Friede M, Reed SG. Adjuvants for malaria vaccines. Parasite Immunol. 2009;31:520-8

4. Coler RN, Goto Y, Bogatzki L, Raman V, Reed SG. Leish-111f, a recombinant polyprotein vaccine that protects against visceral leishmaniasis by elicitation of CD4+ T cells. Infect Immunol. 2007;75:4648-54

5. Coler RN, Reed SG. Second-generation vaccines against leishmaniasis. Trends Parasitol 2005;21:244-9.

6. Garg R, Dube A. Animal models for vaccine studies for visceral leishmaniasis. Indian J Med Res. 2006; 123:439-54.

7. Gicheru MM, Olobo JO, Kariuki TM, Adhiambo C. Visceral leishmaniasis in vervet monkeys: immunological responses during asymptomatic infections. Scand J Immunol. 1995;41:202-8.

8. Gicheru MM, Olobo JO, Anjili CO, Orago AS, Modabber F, Scott P. Vervet monkeys vaccinated with killed Leishmania major parasites and Interleukin-12 develop a type 1 immune response but are not protected against challenge infection. Infect Immun. 2001;69:245-51

9. Kamil AA, Khalil EA, Musa AM, Modabber F, Mukhtar MM, Ibrahim ME, et al. Alumprecipitated autoclaved Leishmania major plus Bacilli Calmette-Guerrin, a candidate vaccine for visceral leishmaniasis: safety, skin-delayed type hypersensitivity response and dose finding in healthy volunteers. Trans R Soc Trop Med Hyg. 2003;97:365-8.

10. Kenney RT, Edelman R. Survey of human-use adjuvants. Expert Rev Vaccines. 2003;2:167-88.

11. Masina S, Gicheru MM, Demotz SO, Fasel NJ. Protection against cutaneous leishmaniasis in outbred Vervet monkeys, using a recombinant histone $\mathrm{H} 1$ antigen. J Infect Dis. 2003; 188:1250-7.

12. Misra A, Dube A, Srivastava B, Sharma P, Srivastava JK, Katiyar JC, et al. Successful vaccination against Leishmania donovani infection in Indian langur using alumprecipitated autoclaved Leishmania major with BCG. Vaccine. 2001;19:3485-92.

13. Mutiso JM, Macharia JC, Gicheru MM. A review of adjuvants for Leishmania vaccine candidates. J Biomed Res; 2010a;24:16-25.
14. Mutiso JM, Macharia JC, Mutisya RM, Taracha E. Subcutaneous immunization against Leishmania major-infection in mice: efficacy of formalin-killed promastigotes combined with adjuvants. Rev Inst Med Trop Sao Paulo. 2010b;52:95-100.

15. Ngure PK, Kimutai A, Ng'ang'a ZW, Rukunga G, Tonui WK. A review of leishmaniasis in Eastern Africa. J Nanjing Med Univ. 2009;23:79-86.

16. Oliveira GA, Wetzel K, Calvo-Calle JM, Nussenzweig R, Schmidt A, Birkett A, et al Safety and enhanced immunogenicity of a hepatitis B core particle Plasmodium falciparum malaria vaccine formulated in adjuvant Montanide ISA 720 in a phase I trial. Infect Immun. 2005; 73:3587-97.

17. Olobo JO, Reid GDF. Mitogenic responses of peripheral blood mononuclear cells of vervet monkeys (Cercopithecus aethiops): apparent role of adherent cells. Am J Primatol. 1990;20:31-6.

18. Pierce MA, Ellis RD, Martin LB, Malkin E, Tierney E, Miura K, et al. Phase 1 safety and immunogenicity trial of the Plasmodium falciparum blood-stage malaria vaccine AMA1-C1/ISA 720 in Australian adults. Vaccine. 2010;28:2236-42.

19. Reed SG, Coler RN, Campos-Neto A. Development of a leishmaniasis vaccine: the importance of MPL. Expert Rev Vaccines. 2003;2:239-52.

20. Ritter U, Korner H. Divergent expression of inflammatory dermal chemokines in cutaneous leishmaniasis. Parasit Immunol 2002; 24:295-301.

21. Skeiky YA, Coler RN, Brannon M, Stromberg E, Greeson K, Craner RT, et al. Protective efficacy of a tandemly linked, multi-subunit recombinant leishmanial vaccine (Leish111f) formulated in MPL adjuvant. Vaccine. 2002;20:3292-303.

22. Toledo H, Baly A, Castro O, Resik S, Laferté J, Rolo F, et al. A phase I clinical trial of a multi-epitope polypeptide TAB9 combined with Montanide ISA 720 adjuvant in non-HIV-1 infected human volunteers. Vaccine. 2001;19:4328-36.

23. Vernacchio L, Bernstein H, Pelton S, Allen C, MacDonald K, Dunn J, et al. Effect of monophosphoryl lipid A (MPL ${ }^{\circledR}$ ) on T-helper cells when administered as an adjuvan with pneumocococcal-CRM ${ }_{197}$ conjugate vaccine in healthy toddlers. Vaccine. 2002;20:3658-67

24. Wu Y, Ellis RD, Shaffer D, Fontes E, Malkin EM, Mahanty S, et al. Phase 1 trial of malaria transmission blocking vaccine candidates Pfs 25 and Pvs 25 formulated with montanide ISA 51. PLos One. 2008;3:e2636.

Received: 06 June 2011

Acccepted: 10 November 2011 